\title{
Vascular endothelial growth factor antagonism restores epithelial barrier dysfunction via affecting zonula occludens proteins
}

\author{
HASAN YUKSEL ${ }^{1}$, OZGE YILMAZ ${ }^{1}$, MERAL KARAMAN ${ }^{2}$, FATIH FIRINCI $^{3}$, \\ AHMET TURKELI $^{1}$, ESRA TOPRAK KANIK ${ }^{1}$ and SEVINC INAN ${ }^{4}$ \\ ${ }^{1}$ Department of Pediatric Allergy and Pulmonology, Medical Faculty, Celal Bayar University, Manisa 45030; \\ ${ }^{2}$ Multidisciplinary Laboratory; ${ }^{3}$ Department of Pediatric Allergy and Immunology, Medical Faculty, Dokuz Eylül University, \\ Izmir 35210; ${ }^{4}$ Department of Histology and Embryology, Medical Faculty, Celal Bayar University, Manisa 45030, Turkey
}

Received July 25, 2014; Accepted April 13, 2015

DOI: $10.3892 / \mathrm{etm} .2015 .2502$

\begin{abstract}
Epithelial barrier dysfunction is important in the pathogenesis of asthma and allergic responses, and is therefore a therapeutic target. The aim of the present study was to investigate the effects of dexamethasone, a classic therapeutic agent, an anti-tumor necrosis factor agent (etanercept), which is used to treat difficult cases of asthma, and an anti-vascular endothelial growth factor (VEGF) agent (bevacizumab), which is an angiogenesis inhibitor, on zonula occludens (ZO) proteins in an experimental asthma model. The experimental model of asthma was developed using intraperitoneal (IP) and inhaled administration of ovalbumin in $38 \mathrm{BALB} / \mathrm{c}$ mice, which were divided into four groups. The control group $(n=6)$ did not receive any treatment, while the four remaining groups ( $\mathrm{n}=8$ per group) received an IP injection of saline, etanercept, bevacizumab or dexamethasone, respectively. Occludin, claudin and junctional adhesion molecule (JAM) were immunohistochemically stained in the left middle lobe samples using an indirect avidin-peroxidase method, after which the staining was semiquantified with $\mathrm{H}$-scores. Statistically significant differences were observed in the occludin, claudin and JAM $\mathrm{H}$-scores among the four groups $(\mathrm{P}<0.001)$. In the untreated asthma, etanercept, bevacizumab and dexamethasone groups, the median H-scores for occludin were 93, 177, 280 and 198, respectively, while the $\mathrm{H}$-scores for claudin were 82, 193.5, 274 and 202.5, respectively, and the median $\mathrm{H}$-scores for JAM were 130, 210, 288 and 210, respectively. Pairwise comparisons revealed that all three $\mathrm{ZO}$ protein $\mathrm{H}$-scores were significantly lower in the saline group when compared with each treatment group. However, the H-scores of the $\mathrm{ZO}$ proteins were not significantly different between
\end{abstract}

Correspondence to: Professor Hasan Yuksel, Department of Pediatric Allergy and Pulmonology, Medical Faculty, Celal Bayar University, 173 Mimarsinan Bulv, Manisa 45030, Turkey

E-mail: hyukselefe@hotmail.com

Key words: asthma, epithelial barrier, occludin, claudin, mouse model the etanercept and dexamethasone groups. Furthermore, the bevacizumab group exhibited higher $\mathrm{H}$-scores for all the proteins compared with the dexamethasone group. Therefore, antagonism of VEGF with bevacizumab restores the epithelial barrier to a greater extent when compared with dexamethasone treatment. This result may be promising for the development of novel therapeutic agents.

\section{Introduction}

Epithelial tissue, in addition to forming a physical barrier to external stimuli, plays a major role in the regulation of the adaptive immune response. The physical barrier function of the epithelium is attained via cell-cell interactions and via impermeable tight junctions (TJs) that are located subapically (1).

Claudins, occludin and junctional adhesion molecules (JAMs) are major constituent proteins of TJs. The claudin family comprises 24 members, and determines the TJ charge selectivity and the location of the paracellular space (2). In addition, human fetal lung cells have been shown to use differential claudin expression to regulate TJ permeability (3). Occludin is an additional protein that is important in barrier formation and regulation (2). JAM-1, which is a member of the immunoglobulin superfamily, has been shown to interact with zonula occludens (ZO)-1, occludin and cingulin, which form the TJ; JAM-1 is hypothesized to affect signal cascades resulting from homophilic and heterophilic adhesion (4). Epithelial barrier function is impaired in individuals with asthma, which is associated with increased permeability to macromolecules, such as allergens. In addition, epithelial barrier impairment in asthmatic bronchi has been shown to be associated with a patchy intercellular distribution of TJs and a decrease in the expression levels of TJ proteins, including ZO-1 and occludin (5).

Tumor necrosis factor (TNF)- $\alpha$ has been shown to reduce claudin expression in a variety of tissues (6-8). However, the mechanism underlying this effect remains unclear with different studies reporting variable results. One study reported that the inflammatory cytokines, TNF- $\alpha$ and interferon- $\gamma$, cause a dose- and time-dependent increase in intestinal epithelial permeability by inducing the redistribution of $\mathrm{TJ}$ 
proteins, including claudin-1, claudin-4 and JAM-2, without causing a significant decrease in their total levels (7). However, an additional study reported that the levels of claudin-1 and claudin-4 did not markedly change, while the downregulation of JAM was observed in a human airway epithelial cell line (9). Furthermore, a previous study reported that TNF- $\alpha$ causes the decreased expression of active occludin molecules, which leads to the disruption of TJ structure in Caco-2 cells (8). However, an additional study reported that TNF- $\alpha$ causes an increase in the levels of active occludin in human airway epithelial cells, which was hypothesized to have occurred due the presence of a leaky junction $(8,9)$. Thus, TNF- $\alpha$ antagonism is hypothesized to improve epithelial barrier integrity.

There is little knowledge with regard to the influence of vascular endothelial growth factor (VEGF) antagonism on epithelial barrier function and TJ structure. The majority of data on the issue are from studies using the retinal endothelium, which have indicated that VEGF exposure leads to a decrease in transendothelial resistance, an increase in vascular permeability, and a decrease in the expression levels of claudin-1 and occludin (10,11). Furthermore, VEGF antagonism has been demonstrated to reverse the decrease in transendothelial resistance and claudin-1 levels induced by VEGF in retinal endothelial cells (12).

Steroid treatment has been shown to improve epithelial barrier integrity, increase transepithelial resistance and lead to a more integrated expression of the TJ proteins, occludin and ZO-1. These effects have been proposed to be mediated through epidermal growth factor receptor phosphorylation (13).

Investigating the effects of different asthma treatments on the epithelial barrier proteins, occludin, claudin and JAM, may identify possible therapeutic alternatives and targets. Therefore, the aim of the present study was to investigate the change in the levels of the TJ proteins, occludin, claudin and JAM, in models of healthy and asthmatic mice, and to compare the influence of TNF and VEGF inhibition and steroid treatment on these changes.

\section{Materials and methods}

Experimental animals. A total of $38 \mathrm{BALB} / \mathrm{c}$ mice (age, 6-8 weeks; weight, 18-20 g) were used in the experiment. The mice were housed in hygienic macrolene cages in air-conditioned rooms on a 12-h light/dark cycle. Food and water were provided ad libitum. The animals were maintained at a constant temperature of $22^{\circ} \mathrm{C}$ in the Experimental Animal Unit of Dokuz Eylul University (Izmir, Turkey).

This study was conducted in the Dokuz Eylul University multidisciplinary laboratory and approved by the university ethical board. Furthermore, the study was conducted in accordance with the recommendations outlined in the Guidelines for the Care and Use of Experimental Animals (14).

Study design. The study comprised five groups of mice. Control group $(n=6)$ mice were not exposed to ovalbumin (OVA) or any other medication. A chronic asthma model was established in the other four groups ( $n=8$ per group) through application of intraperitoneal (IP) and inhaled OVA exposure. Each of these latter four groups received as follows: IP saline injection once per week for 2 weeks; IP injection of etanercept (Wyeth Europa Ltd, Maidenhead, UK) at $0.01 \mathrm{mg} /$ dose $(0.5 \mathrm{mg} / \mathrm{kg})$ twice per week for two weeks; IP injection of bevacizumab (Avastin; Roche Diagnostics, Basel, Switzerland) at $0.15 \mathrm{mg}(5 \mathrm{mg} / \mathrm{kg})$ once per week for two weeks; or $1 \mathrm{mg} / \mathrm{kg} / \mathrm{dose}$ IP injection of dexamethasone (Dekort; Deva Holding AS, Istanbul, Turkey) for 7 days during the final week of OVA inhalation.

Sensitization procedure and inhalation exposure. A chronic murine model of asthma, as described by Temelovski et al (15), was successfully established. An injection of $10 \mu \mathrm{g}$ OVA (grade V, $\geq 98 \%$ pure; Sigma-Aldrich, St. Louis, MO, USA), at a volume of $0.1 \mathrm{ml}$, was administered (IP) to the BALB/c mice (with the exception of the Group 1 mice) at days 21 and 7 prior to OVA inhalation, with alum as the adjuvant. Consequently, the mice were exposed to a $2.5 \%$ OVA aerosol in sterile saline for $30 \mathrm{~min} /$ day for three days/week for a total of eight weeks, starting on day 21 of the experiment. Exposure was conducted using a whole body inhalation exposure system in a plexiglass

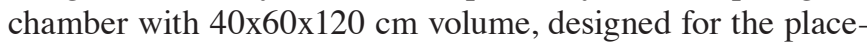
ment of cages. A particle concentration of $10-20 \mathrm{mg} / \mathrm{m}^{3}$ was achieved, and $\geq 80 \%$ of the particles were expected to have a diameter of $\leq 4 \mu \mathrm{m}$, due to the characteristics of the system used.

The mice were anesthetized by an IP injection of ketamine hydrochloride $(200 \mathrm{mg} / \mathrm{kg})$ at day 1 following the administration of the final medication, after which the mice were sacrificed via exsanguination cardiac arrest.

Histopathological and immunohistochemical evaluations. Formalin-fixed, paraffin-embedded lung samples collected from the control and experimental mice were prepared by routine paraffin-embedding procedures. In brief, tissue samples were fixed in $10 \%$ formalin in phosphate buffer $(\mathrm{pH} 7.4)$ for $48 \mathrm{~h}$, dehydrated in a graded series of ethanol $(70 \%, 80 \%$, $90 \%$ and absolute ethanol), cleared in xylene and embedded in paraffin. The paraffin blocks were serially sectioned using a Leica RM2245 rotary microtome (Leica Microsystems Gmbh, Wetzlar, Germany) at a thickness of $5 \mu \mathrm{m}$, and prepared for histochemical and immunohistochemical staining. For histological assessment, the sections were stained with hematoxylin and eosin.

For immunohistochemical staining, the sections were incubated at $60^{\circ} \mathrm{C}$ overnight, cleared in xylene for $30 \mathrm{~min}$, and washed with decreasing concentrations of ethanol, followed by washing with distilled water. After incubation in phosphate-buffered saline PBS (P4417; Sigma-Aldrich) for $10 \mathrm{~min}$, the sections were treated with 2\% trypsin (TSS155; Scytek Laboratories, Inc., Logan, UT, USA) at $37^{\circ} \mathrm{C}$ for $10 \mathrm{~min}$ and washed with PBS. The sections were delineated with a Dako pen (s2002; Dako, Glostrup, Denmark), incubated in a solution of 3\% hydrogen peroxide (K31355100; Merck, Darmstadt, Germany) for $15 \mathrm{~min}$ to inhibit endogenous peroxidase activity, and washed with PBS. Subsequently, primary antibodies were added and the sections were incubated in a humidified chamber at $4^{\circ} \mathrm{C}$ overnight. The following primary antibodies were used at a 1:100 dilution: Claudin-1 antibody (A-9; sc-166338; mouse monoclonal IgG2b; Santa Cruz Biotechnology, Inc., Santa Cruz, CA, USA), occludin antibody (sc-133256; mouse monoclonal IgG2b; Santa Cruz Biotechnology, Inc.) and JAM-A 
Table I. Occludin, claudin and JAM H-scores in the study groups.

\begin{tabular}{lccc}
\hline Study groups & Occludin H-score & Claudin H-score & JAM H-score \\
\hline Non-asthmatic control & $286(269-304)$ & $306(300-320)$ & $274(259-304)$ \\
Untreated asthma & $93(78-130.5)$ & $82(76.5-108)$ & $130(106.3-160)$ \\
Etanercept & $177(169.5-245)$ & $193.5(186-238.3)$ & $210(184.5-236)$ \\
Bevacizumab & $280(239.5-310)$ & $274(229.8-306)$ & $288(243-304)$ \\
Dexamethasone & $198(180-220.5)$ & $202.5(195-210)$ & $210(186-231)$ \\
P-value & $\mathrm{P}<0.001$ & $\mathrm{P}<0.001$ & $\mathrm{P}<0.001$ \\
\hline
\end{tabular}

Values are expressed as the median (25-75 percentile). P-values were calculated using the Kruskal-Wallis test. JAM, junctional adhesion molecule.

antibody (H-80; sc-25629; rabbit polyclonal IgG; Santa Cruz Biotechnology, Inc.).

Following removal of the primary antibodies, the sections were washed with PBS three times for 5 min each and incubated with a biotinylated secondary antibody for $30 \mathrm{~min}$, using a Histostain ${ }^{\circledR}$-Plus-Peroxidase kit (85-9043; Invitrogen Life Technologies, Carlsbad, CA, USA). Subsequently, the sections were incubated with streptavidin conjugated to horseradish peroxidase in PBS for 30 min, using a Histostain ${ }^{\circledR}$-Plus Bulk kit, Invitrogen 2nd Generation, labeled-(strept)avidin-biotin Detection System (85-8943; Invitrogen Life Technologies). The sections were subsequently washed with PBS three times and incubated for 5 min with 3,3'-diaminobenzidine (00-2020; Invitrogen Life Technologies) as a chromogen. After washing with distilled water, the sections were counterstained with Mayer's hematoxylin solution (\#02274390059; J.T. Barker, Deventer, The Netherlands), washed with tap water, dehydrated through a graded series of alcohol and cleared in xylene. The sections were mounted with Entellan mounting medium (HX265767; Merck Millipore, Darmstadt, Germany) and evaluated under a light microscope (BX40; Olympus Corporation, Tokyo, Japan). Control samples were processed in an identical manner, with the exception that $\operatorname{IgG}$ was used instead of a primary antibody.

The distribution of immunohistochemical intensities of the primary antibodies was scored as mild $(+)$, moderate $(++)$, strong $(+++)$ and very strong $(++++)$ brown staining. Staining intensity was graded semiquantitatively using $\mathrm{H}$-scores, which were calculated using the following equation: $\mathrm{H}$-score $=\Sigma \mathrm{Pi}$ $(i+1)$, where $i$ was equal to the intensity of immunohistochemical staining with a value of 1-4, and Pi was the percentage of epithelial cells stained with each intensity, varying between $0-100 \%$ (16).

Statistical analysis. Statistical analysis was performed using SPSS 15.0 software (SPSS, Inc., Chicago, IL, USA). The Kruskal-Wallis non-parametric test was used to compare the results among all four treatment groups, while the Mann-Whitney U test was used for pairwise comparison of the groups. $\mathrm{P}<0.05$ was considered to indicate a statistically significant difference. The Bonferroni correction was applied for the post-hoc analysis results, where $\mathrm{P}<0.005$ was regarded as a statistically significant difference for the two group comparisons.

\section{Results}

Occludin H-scores among the groups. The difference in the occludin H-scores was determined to be statistically significant when the five groups were compared $(\mathrm{P}<0.001)$. In the non-asthmatic control group, the median occludin $\mathrm{H}$-score was 286 , which was significantly higher compared with the untreated asthma, etanercept and dexamethasone groups (93, 177 and 198 respectively; $\mathrm{P}=0.002$ for all). By contrast, the difference in the occludin $\mathrm{H}$-scores between the bevacizumab group and non-asthmatic control group was not statistically significant ( 280 vs. 286 respectively; $\mathrm{P}=0.60$; Table I).

Occludin H-scores in the untreated asthma group were significantly lower compared with the etanercept, bevacizumab and dexamethasone groups $(\mathrm{P}=0.005, \mathrm{P}=0.001$ and $\mathrm{P}=0.004$, respectively).

Comparison of the treatment groups with each other revealed that the differences in the occludin H-scores between the etanercept group and the bevacizumab or dexamethasone groups were not statistically significant $(\mathrm{P}=0.01$ and $\mathrm{P}=0.79$, respectively). By contrast, the bevacizumab group had a significantly higher median occludin H-score when compared with the dexamethasone group ( 280 vs. 198 respectively; $\mathrm{P}=0.001$; Table I and Figs. 1 and 2).

Claudin H-scores among the groups. Median claudin H-scores were significantly different among the groups. In the non-asthmatic control group, the claudin $\mathrm{H}$-score was significantly higher compared with the untreated asthma and dexamethasone groups (306 vs. 82 and 202.5, respectively; $\mathrm{P}=0.002$ for the two comparisons); however, there were no statistically significant differences when comparing the non-asthmatic control with the etanercept or bevacizumab groups (193.5 and 274, respectively; $\mathrm{P}=0.03$ and $\mathrm{P}=0.11$ respectively; Table I). The untreated asthma group had a significantly lower claudin $\mathrm{H}$-score compared with the three treatment groups (82 vs. 193.5 in the etanercept, 274 in the bevacizumab and 202.5 in the dexamethasone groups; $\mathrm{P}=0.004, \mathrm{P}=0.001$ and $\mathrm{P}=0.002$, respectively; Table I).

Comparison of the three treatment groups with each other revealed that the dexamethasone group had similar claudin $\mathrm{H}$-scores compared with the etanercept group, but lower claudin $\mathrm{H}$-scores when compared with the bevacizumab group $(\mathrm{P}=0.75$ and $\mathrm{P}=0.001$, respectively). Similarly, etanercept treat- 


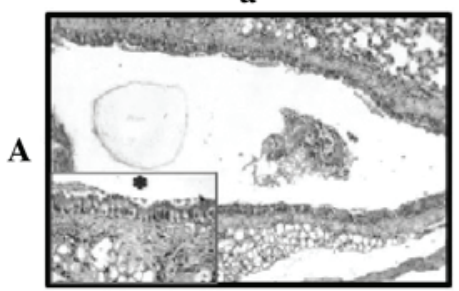

B

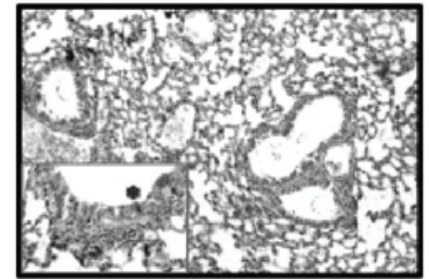

C

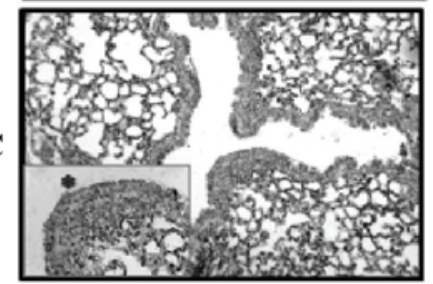

D

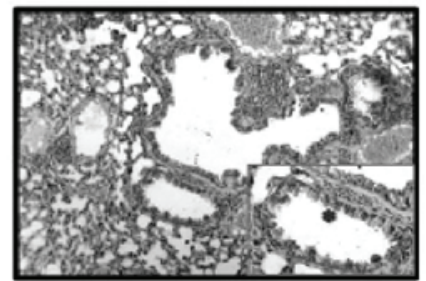

$\mathbf{E}$

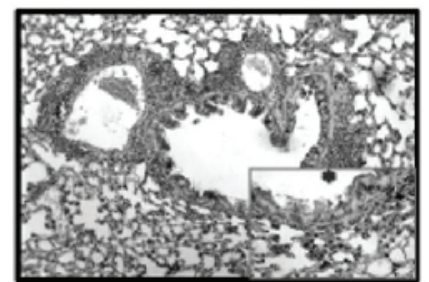

b
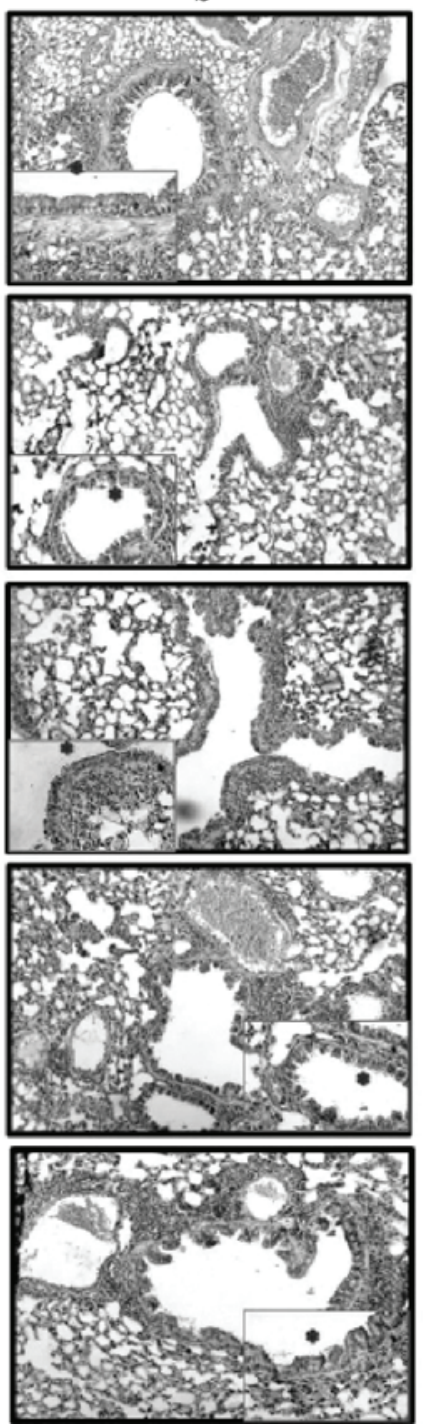
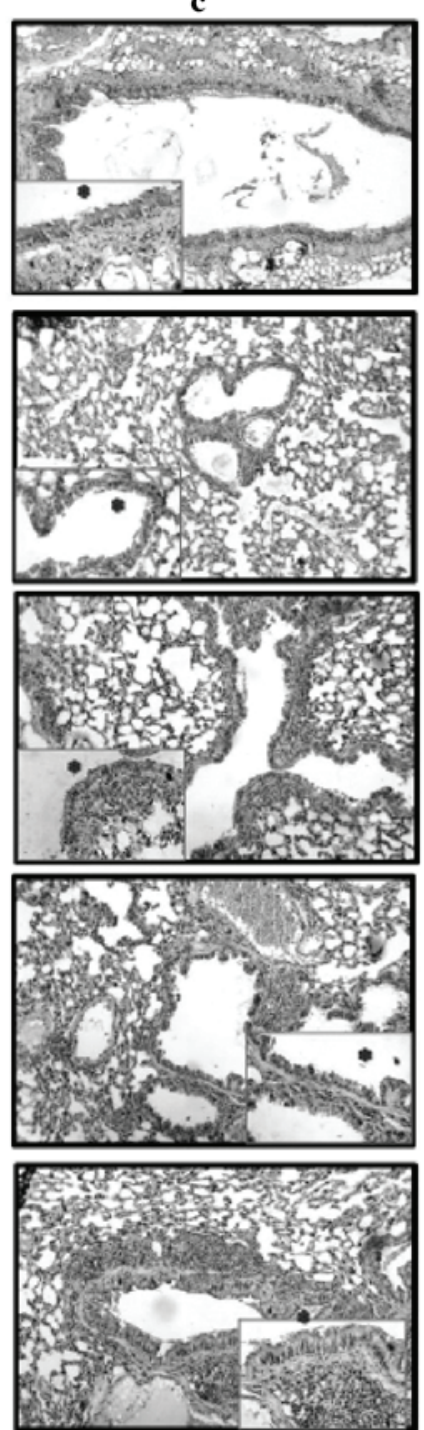

Figure 1. Anti-occludin, anti-claudin and anti-junctional adhesion molecule (JAM) staining of the five groups (original magnification, $\mathrm{x} 100$; insert, $\mathrm{x} 400$ ). (A) Asthmatic control, (B) untreated asthma, (C) etanercept, (D) dexamethasone and (E) bevacizumab groups. Within these: (a) Occludin, (b) claudin; and (c) JAM immunoreactivity. "Immunoreactivity. Ep, epithelium.

ment was not significantly different from the bevacizumab treatment in terms of the levels of claudin $(\mathrm{P}=0.05$; Table I and Figs. 1 and 3).

JAM H-scores among the groups. The median JAM H-score in the non-asthmatic control group was 274, which was significantly higher compared with the untreated asthma and dexamethasone groups ( $\mathrm{P}=0.002$ for both). However, no statistically significant difference was detected when this was compared with the etanercept and bevacizumab groups $(\mathrm{P}=0.01$ and $\mathrm{P}=0.89$, respectively). The untreated asthma group had a median JAM H-score of 130 , which was significantly lower compared with all three treatment groups $(\mathrm{P}=0.002$, $\mathrm{P}=0.001$ and $\mathrm{P}=0.001$ for the etanercept, bevacizumab and dexamethasone groups, respectively; Table I).

Comparisons between the three treatment groups revealed that the etanercept group had similar JAM H-scores when compared with the bevacizumab and dexamethasone groups $(\mathrm{P}=0.007$ and $\mathrm{P}=0.87$ respectively). However, the bevacizumab group had significantly higher JAM H-scores compared with the dexamethasone group ( $\mathrm{P}=0.004$; Table I and Figs 1 and 4).

\section{Discussion}

The present study demonstrated that the levels of the TJ proteins, occludin, claudin and JAM, were decreased in a murine model of asthma. Immunohistochemical evaluation of the expression levels of occludin, claudin and JAM following the different asthmatic treatment modalities revealed that treatment with steroids, as well as TNF and VEGF antagonists, increased the levels of the proteins when compared with untreated asthma group. The only difference among the three treatment groups was between dexamethasone and bevacizumab (VEGF antagonism), which was in favor of the latter. Furthermore, TNF and VEGF antagonism resulted in levels of claudin and JAM that were similar to the non-asthmatic control, while only VEGF antagonism led to levels of occludin that were similar to the non-asthmatic control. 


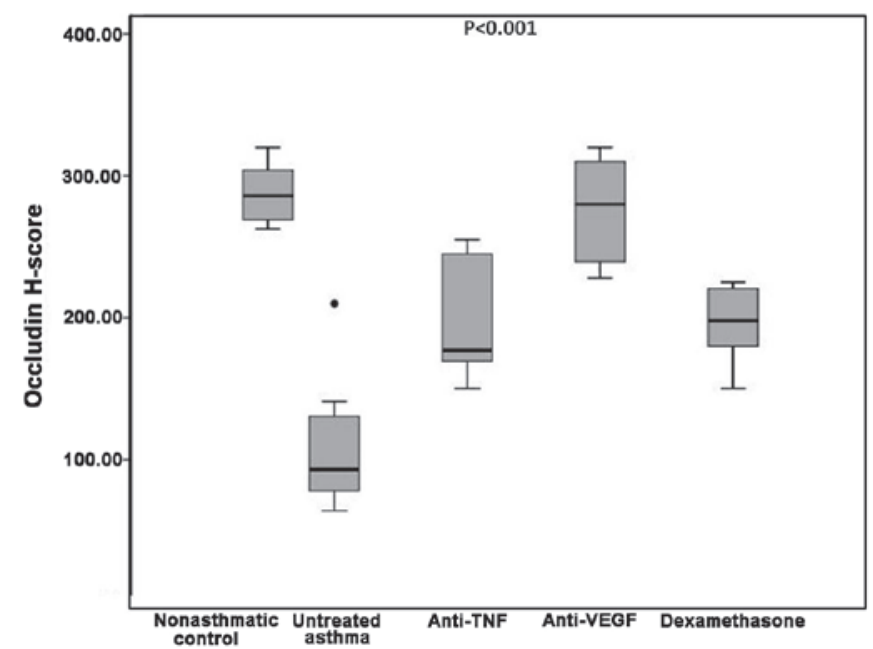

Figure 2. Boxplot of the occludin $\mathrm{H}$-scores in the various groups. TNF, tumor necrosis factor; VEGF, vascular endothelial growth factor.

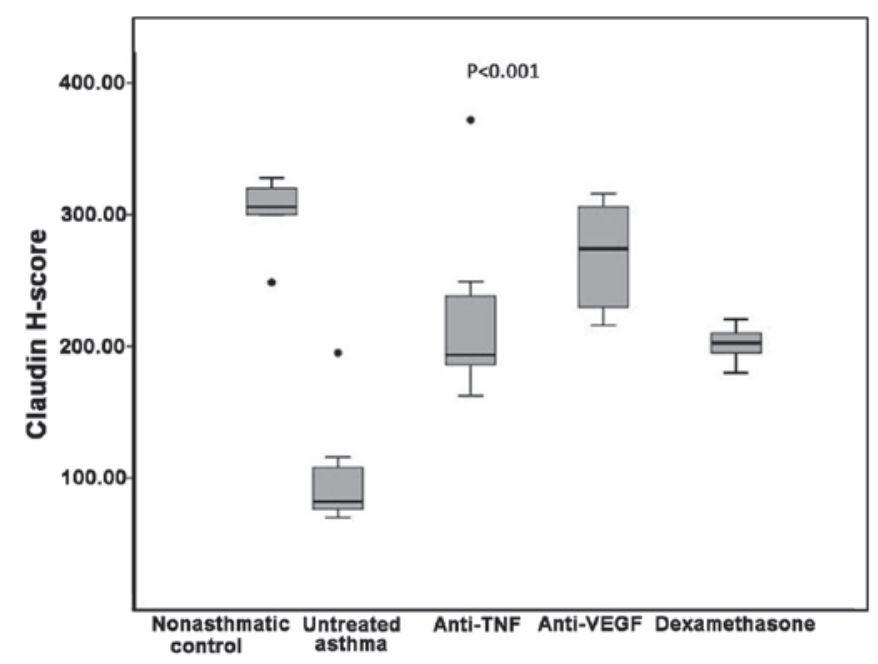

Figure 3. Boxplot of the claudin H-scores in the various groups. TNF, tumor necrosis factor; VEGF, vascular endothelial growth factor.

Asthma is a chronic inflammatory disease of the airways that originally was attributed to a deviated adaptive immune system, primarily affecting T helper 2 lymphocytes; however, novel pathogenetic results have indicated the role of the innate immune system, including the epithelium, in the pathogenesis of asthma (17). Under physiological conditions, the airway epithelium forms a barrier to inhaled antigens and allergens; however, this barrier is impaired in individuals with asthma and the permeability to inhaled allergens and pollutants is increased, which is hypothesized to be the mechanism underlying the development of asthma in certain atopic individuals $(18,19)$. Concordantly, the present study demonstrated significantly decreased levels of the TJ proteins, occludin, claudin and JAM, in the bronchial epithelium of the asthmatic mice, as compared with the non-asthmatic mice, indicating their role in the pathogenesis of asthma.

Epithelial barrier function is predominantly controlled by TJs that constitute a continuous structure between the apical and basolateral membranes, which regulate the exchange of substances between intracellular and extracellular

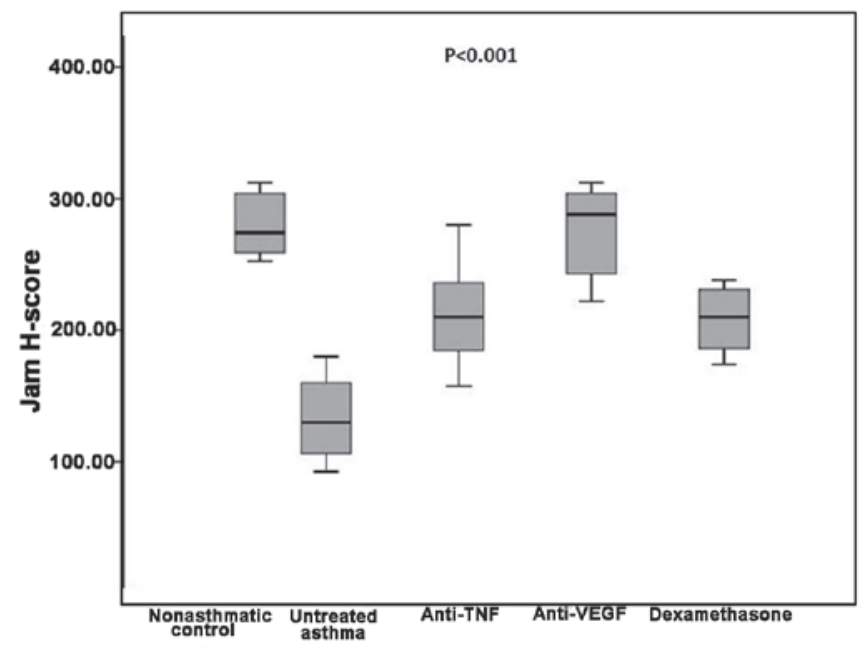

Figure 4. Boxplot of the JAM H-scores in the various groups. TNF, tumor necrosis factor; VEGF, vascular endothelial growth factor; JAM, junctional adhesion molecule.

environments. Occludin, claudin and JAM-1 are the main components controlling the barrier function of TJs through sealing the paracellular space. Occludin copolymerases into claudin-based TJ strands; however, the protein is not required for the formation of TJ strands, but for the regulation of permeability and transepithelial resistance. The claudin family, which comprises 24 members, forms the backbone of the TJ structure and is considered to play a role in the formation of the ion selective pores within the TJ strands. JAMs belong to the immunoglobulin superfamily, and are not part of the TJ strands; however, these proteins interact with TJ proteins, including ZO-1 and cingulin. JAM 1 is hypothesized to play a role in the propagation of signals as a result of homophilic and heterophilic adhesion to other molecules (4). The aim of the current study was to investigate the changes in the levels of these three major proteins, which play a role in the formation of the epithelial barrier, in a mouse model of asthma subjected to different treatment conditions, in an attempt to identify potential therapeutic targets and agents.

Glucocorticoids have been shown to increase endothelial barrier properties by increasing the gene expression levels of occludin and claudin-5 in the retinal endothelium (20). Similarly, the results of the current study revealed increased levels of occludin, claudin and JAM in a mouse model of asthma treated with steroids. These observations indicate the role of conventional steroid treatment in epithelial repair and barrier improvement in asthma.

The role of VEGF has been commonly studied in models of diabetic retinopathy and retinal endothelial cells $(11,21)$. Considering the important role of VEGF in asthma and remodeling, the current study aimed to investigate the effects of VEGF antagonism on bronchial epithelial barrier function (22). VEGF has been observed to cause the phosphorylation of occludin and a decrease in the level of occludin in retinal endothelial cells in diabetic retinopathy, subsequently increasing endothelial permeability $(11,22)$. This finding is concordant with the present results that demonstrated an increase in the level of occludin in the asthmatic mice treated with bevacizumab, resulting in a level that was similar to the 
non-asthmatic control mice. Comparison of VEGF antagonistic treatment with conventional steroid treatment in the asthmatic mice revealed a higher level of bronchial epithelial occludin in the former group. Furthermore, VEGF antagonism has been shown to increase transendothelial electrical resistance in bovine retinas and reverse VEGF-induced loss of claudin-1 protein. In addition, VEGF antagonists have been shown to cause the colocalization of claudin- 1 and claudin- 5 to the plasma membrane, supporting a stable barrier function (12). The present study detected that the level of claudin in the bronchial epithelium increased with VEGF antagonistic treatment in a murine asthma model, resulting in levels that were similar to those of the non-asthmatic controls. Furthermore, the increase in the level of occludin observed with VEGF antagonism was significantly more prominent compared with conventional steroid treatment. The influence of VEGF antagonism on the levels of bronchial JAM was also investigated. JAM levels were shown to increase to a similar level to that observed in the non-asthmatic mice, and this effect was significantly higher compared with the conventional steroid treatment. Overall, VEGF antagonistic treatment appeared to reverse the decrease in the levels of the epithelial barrier TJ proteins, occludin, claudin and JAM, in a mouse model of asthma, and this effect was more pronounced when compared with conventional steroid treatment. Thus, VEGF may be a potential therapeutic target for improving epithelial barrier dysfunction in asthma.

TNF- $\alpha$ expression is known to result in a decrease in the levels of occludin and claudin, and the redistribution of p120 catenin and E-cadherin, which subsequently compromises the properties of TJs and adherens junctions. In turn, the epithelial barrier function is compromised in different tissues, including the brain and lungs (6,23). Subsequently, a decrease in transepithelial electrical resistance occurs, and increased epithelial permeability in asthmatic air-liquid interface cultures has been observed (23). TNF- $\alpha$ is not only effective on bronchial epithelial TJs, but also on other tissues, including retinal epithelial cells, where the cytokine increases the levels of matrix metalloproteinases, which in turn degrade claudin-1 and occludin, increasing permeability (24). In concordance with these previously reported effects of TNF- $\alpha$, antagonism of TNF- $\alpha$ in the present mouse model of asthma resulted in an increase in the levels of occludin and claudin in the bronchial epithelium. Furthermore, the level of an additional TJ protein, JAM, was also shown to increase with TNF- $\alpha$ antagonistic treatment in asthma. These effects of TNF- $\alpha$ antagonistic treatment appeared to be similar to conventional steroid treatment, but inferior to VEGF antagonistic treatment. Therefore, TNF- $\alpha$ antagonistic treatment in steroid-resistant cases of asthma may not be expected to provide additional therapeutic effects on bronchial epithelial barrier function.

One of the main limitations of the present study was the use of the semiquantitative immunohistochemical H-scores. In an attempt to avoid potential bias, the histologist was blinded to the groups when scoring. Furthermore, the use of a mouse model limits the generalization to humans. However, mouse models of asthma are generally accepted to correlate well with clinical human results (25). Finally, the use of animal models precludes the prediction of side effects when these agents are used in humans; however, dexamethasone and etanercept are already widely administered. With regard to bevacizumab, further clinical research is required in order to propose any recommendations for human administration.

The major contribution of the present study to the current literature is the comparison of the influence of different therapeutic agents on epithelial barrier proteins, which are of current interest in investigations into the pathogenesis of asthma. Furthermore, the effect of VEGF inhibition on the bronchial epithelium in asthma has not previously been studied extensively.

In conclusion, the present study demonstrated that the epithelial barrier proteins, occludin, claudin and JAM, are significantly decreased in mouse models of asthma, which results in the impairment of epithelial barrier function. Conventional treatment with steroids was shown to increase the levels of these proteins to a similar degree as TNF- $\alpha$ antagonistic treatment, which subsequently raised the question of the efficacy of TNF- $\alpha$ antagonistic treatment on barrier enhancement in steroid-resistant cases. VEGF antagonists may be potential novel therapeutic agents, since treatment with bevacizumab was shown to increase the expression levels of the bronchial epithelium TJ proteins. However, further clinical research investigating the in vivo efficacy and side-effect profile of bevacizumab in cases of asthma is required prior to the establishment of specific recommendations.

\section{References}

1. Holgate ST: Epithelium dysfunction in asthma: J Allergy Clin Immunol 120: 1233-1244, 2007.

2. Marchiando AM, Graham WV and Turner JR: Epithelial barriers in homeostasis and disease. Annu Rev Pathol 5: 119-144, 2010.

3. Daugherty BL, Mateescu M, Patel AS, Wade K, Kimura S, Gonzales LW, Guttentag S, Ballard PL and Koval M: Developmental regulation of claudin localization by fetal alveolar epithelial cells. Am J Physiol Lung Cell Mol Physiol 287: L1266-L1273, 2004.

4. Förster C: Tight junctions and the modulation of barrier function in disease. Histochem Cell Biol 130: 55-70, 2008.

5. Xiao C, Puddicombe SM, Field S, Haywood J, Broughton-Head V, Puxeddu I, Haitchi HM, Vernon-Wilson E, Sammut D, Bedke N, Cremin C, Sones J, Djukanović R, Howarth PH, Collins JE, Holgate ST, Monk P and Davies DE: Defective epithelial barrier function in asthma. J Allergy Clin Immunol 128: 549-556, 2011.

6. Aslam M, Ahmad N, Srivastava R and Hemmer B: TNF- $\alpha$ induced $\mathrm{NF} \kappa \mathrm{B}$ signaling and p65 (RelA) overexpression repress Cldn 5 promoter in mouse brain endothelial cells. Cytokine 57: 269-275, 2012.

7. Bruewer M, Luegering A, Kucharzik T, Parkos CA, Madara JL, Hopkins AM and Nusrat A: Proinflammatory cytokines disrupt epithelial barrier function by apoptosis-independent mechanisms. J Immunol 171: 6164-6172, 2003.

8. Cui W, Li LX, Sun CM, Wen Y, Zhou Y, Dong YL and Liu P: Tumor necrosis factor alpha increases epithelial barrier permeability by disrupting tight junctions in Caco-2 cells. Braz J Med Biol Res 43: 330-337, 2010.

9. Coyne CB, Vanhook MK, Gambling TM, Carson JL, Boucher RC and Johnson LG: Regulation of airway tight junctions by proinflammatory cytokines. Mol Biol Cell 13: 3218-3234, 2002.

10. Deissler HL, Deissler H, Lang GK and Lang GE: VEGF but not PIGF disturbs the barrier of retinal endothelial cells. Exp Eye Res 115: 162-171, 2013.

11. Antonetti DA, Barber AJ, Khin S, Lieth E, Tarbell JM and Gardner TW; Penn State Retina Research Group: Vascular permeability in experimental diabetes is associated with reduced endothelial occludin content: Vascular endothelial growth factor decreases occludin in retinal endothelial cells. Diabetes 47 : 1953-1959, 1998 
12. Deissler HL, Deissler H and Lang GE: Actions of bevacizumab and ranibizumab on microvascular retinal endothelial cells: Similarities and differences. Br J Ophthalmol 96: 1023-1028, 2012.

13. Sekiyama A, Gon Y, Terakado M, Takeshita I, Kozu Y,Maruoka S, Matsumoto K and Hashimoto S: Glucocorticoids enhance airway epithelial barrier integrity. Int Immunopharmacol 12: 350-357, 2012.

14. National Research Council: Committee for the Update of the Guide for the Care and Use of Laboratory Animals. Guide for the Care and Use of Laboratory Animals. 8th edition. Washington (DC), USA: National Academies Press, 2011

15. Temelkovski J, Hogan SP, Shepherd DP, Foster PS and Kumar RK: An improved murine model of asthma: Selective airway inflammation, epithelial lesions and increased methacholine responsiveness following chronic exposure to aerosolised allergen. Thorax 53: 849-856, 1998.

16. Yuksel H, Yilmaz O, Baytur YB and Ozbilgin K: Prenatal administration of granulocyte-macrophage colony-stimulating factor increases mesenchymal vascular endothelial growth factor expression and maturation in fetal rat lung. Exp Lung Res 34 550-558, 2008.

17. Lambrecht BN and Hammad H: Asthma: The importance of dysregulated barrier immunity. Eur J Immunol 43: 3125-3137, 2013.

18. Holgate ST: Pathogenesis of asthma. Clin Exp Allergy 38: 872-897, 2008

19. Holgate ST: The sentinel role of the airway epithelium in asthma pathogenesis. Immunol Rev 242: 205-219, 2011.
20. Felinski EA, Cox AE, Phillips BE and Antonetti DA: Glucocorticoids induce transactivation of tight junction genes occludin and claudin-5 in retinal endothelial cells via a novel cis-element. Exp Eye Res 86: 867-878, 2008.

21. Harhaj NS, Felinski EA, Wolpert EB, Sundstrom JM, Gardner TW and Antonetti DA: VEGF activation of protein kinase $\mathrm{C}$ stimulates occludin phosphorylation and contributes to endothelial permeability. Invest Ophthalmol Vis Sci 47: 5106-5115, 2006.

22. Yuksel H, Kose C, Yilmaz O, Ozbilgin K, Degirmenci PB, Pinar E and Kirmaz C: Increased expression of tissue vascular endothelial growth factor and foetal liver kinase-1 receptor in seasonal allergic rhinitis and relevance to asthma component. Clin Exp Allergy 37: 1183-1188, 2007.

23. Hardyman MA, Wilkinson E, Martin E, Jayasekera NP, Blume C, Swindle EJ, Gozzard N, Holgate ST, Howarth PH, Davies DE and Collins JE: TNF- $\alpha$-mediated bronchial barrier disruption and regulation by src-family kinase activation. J Allergy Clin Immunol 132: 665-675, 2013.

24. Yamada $H$, Yoneda M, Inaguma S, Watanabe D, Banno S, Yoshikawa K, Mizutani K, Iwaki M and Zako M: Infliximab counteracts tumor necrosis factor- $\alpha$-enhanced induction of matrix metalloproteinases that degrade claudin and occludin in non-pigmented ciliary epithelium. Biochem Pharmacol 85: 1770-1782, 2013.

25. Arnett HA and Viney JL: Considerations for the sensible use of rodent models of inflammatory disease in predicting efficacy of new biological therapeutics in the clinic. Adv Drug Deliv Rev 59: 1084-1092, 2007. 